\title{
Identification of Root-Secreted Compounds Involved in the Communication Between Cucumber, the Beneficial Bacillus amyloliquefaciens, and the Soil-Borne Pathogen Fusarium oxysporum
}

\author{
Yunpeng Liu, ${ }^{1}$ Lin Chen, ${ }^{2}$ Gengwei Wu, ${ }^{2}$ Haichao Feng, ${ }^{2}$ Guishan Zhang, ${ }^{1}$ Qirong Shen, ${ }^{2}$ and \\ Ruifu Zhang ${ }^{1,2}$ \\ ${ }^{1}$ Key Laboratory of Microbial Resources Collection and Preservation, Ministry of Agriculture, Institute of Agricultural Resources \\ and Regional Planning, Chinese Academy of Agricultural Sciences, Beijing 100081, P.R. China; and ${ }^{2}$ Jiangsu Key Lab and \\ Engineering Center for Solid Organic Waste Utilization, National Engineering Research Center for Organic-based Fertilizers, \\ Nanjing Agricultural University, Nanjing, 210095, P.R. China
}

Accepted 12 December 2016.

\begin{abstract}
Colonization of plant growth-promoting rhizobacteria (PGPR) is critical for exerting their beneficial effects on the plant. Root exudation is a major factor influencing the colonization of both PGPR and soil-borne pathogens within the root system. However, the tripartite interaction of PGPR, plant roots, and soil-borne pathogens is poorly understood. We screened root exudates for signals that mediate tripartite interactions in the rhizosphere. In a split-root system, we found that root colonization of PGPR strain Bacillus amyloliquefaciens SQR9 on cucumber root was significantly enhanced by preinoculation with SQR9 or the soil-borne pathogen Fusarium oxysporum f. sp. cucumerinum, whereas root colonization of $F$. oxysporum $f$. sp. cucumerinum was reduced upon preinoculation with SQR9 or F. oxysporum f. sp. cucumerinum. Root exudates from cucumbers preinoculated with SQR9 or $F$. oxysporum f. sp. cucumerinum were analyzed and 109 compounds were identified. Correlation analysis highlighted eight compounds that significantly correlated with root colonization of SQR9 or F. oxysporum f. sp. cucumerinum. After performing colonization experiments with these chemicals, raffinose and tryptophan were shown to positively affect the root colonization of F. oxysporum f. sp. cucumerinum and SQR9, respectively. These results indicate that cucumber roots colonized by $F$. oxysporum $f$. sp. cucumerinum or SQR9 increase root secretion of tryptophan to strengthen further colonization of SQR9. In contrast, these colonized cucumber roots reduce raffinose secretion to inhibit root colonization of $\boldsymbol{F}$. oxysporum f. sp. cucumerinum.
\end{abstract}

Agricultural production is facing a challenge from plant diseases, such as soil-borne diseases, that have been reported to lead to sharp decreases in food production (Qiu et al. 2012; Ye et al. 2004). To date, wilt disease caused by Fusarium oxysporum f. sp. сисиmerinum has seriously affected cucumber production worldwide. However, some plant growth-promoting rhizobacteria (PGPR)

Corresponding author: R. Zhang; E-mail: zhangruifu@ @aas.cn; Telephone: +86-10-82108634; Fax: +86-10-82108683

*The $\boldsymbol{e}$-Xtra logo stands for "electronic extra" and indicates that three supplementary figures and three supplementary tables are published online.

(c) 2017 The American Phytopathological Society have been shown to be effective at suppressing soil-borne pathogens and improving crop production (Wang et al. 2015).

Root colonization is a prerequisite for both soil-borne pathogens and PGPR to exert their pathogenic and biocontrol function due to the effects of root exudation (Raaijmakers et al. 2009). However, both the composition and the amount of root exudates are dependent on various factors, such as plant species and microorganism status in the rhizosphere (Rudrappa et al. 2008). It is widely accepted that root exudates comprise two different classes of compounds: the low molecular weight compounds, including a variety of organic acids, sugars, and the secondary metabolites, and high molecular weight compounds, including proteins and polysaccharides (Badri and Vivanco 2009; Bais et al. 2006; Lugtenberg et al. 1999). The root colonization of microbes is a major factor affecting secretion from roots (Badri and Vivanco 2009; Bais et al. 2006; Rudrappa et al. 2008). Nevertheless, how infection or root colonization of rhizosphere microbes affects root exudation is not well understood, in particular the tripartite interactions of PGPR, root exudates, and soil-borne pathogens.

Bacillus amyloliquefaciens SQR9 was isolated from the rhizosphere of a cucumber plant and has strong antagonistic activity against $F$. oxysporum f. sp. cucumerinum (Cao et al. 2012; Zhang et al. 2008). In a previous study, we found that infection with $F$. oxysporum f. sp. cucumerinum increased cucumber root secretion of citric acid and fumaric acid, which enhanced the root colonization of SQR9 (Liu et al. 2014). However, how the root colonization of SQR9 affects the behavior of $F$. oxysporum f. sp. cucumerinum in the rhizosphere is still unknown. Moreover, the signals from root exudates involved in the interaction of PGPR, plant roots, and soil-borne pathogens have not been comprehensively explored.

In this study, a split-root system in which plant roots could be divided into two parts and treated separately was designed and used. To explore the interaction between $F$. oxysporum f. sp. cucumerinum, cucumber, and SQR9, the influence of previous root inoculation by SQR9, $F$. oxysporum f. sp. cucumerinum, the PGPR strain Pseudomonas brassicacearum J12, or the nonPGPR strain Bacillus subtilis 168 on the subsequent colonization of SQR9 was investigated in the split-root system without actual contact between the two inoculants (Fig. 1, experiment A). The use of strains J12 and 168 was to test whether the effect of SQR9 is common in PGPR. Separately, its influence on 
subsequent colonization of $F$. oxysporum $\mathrm{f}$. sp. cucumerinum was investigated as a parallel experiment (Fig. 1, experiment B). Meanwhile, as a separate experiment, after the previous root inoculation of the microbes in the left side, root exudates collection and analysis were performed in the right side (Fig. 1, experiment C). Subsequently, all detected compounds in root exudates were correlated with the root colonization of SQR9 or $F$. oxysporum f. sp. cucumerinum, to search the potential signals involved in the tripartite interaction of $F$. oxysporum f. sp. cucumerinum, cucumber, and SQR9, and further verification was performed.

\section{RESULTS}

\section{Systemic effect of preinoculation}

on the subsequent root colonization

of SQR9 and F. oxysporum f. sp. cucumerinum.

The influence of previous root colonization by different microbes such as Bacillus amyloliquefaciens SQR9 or the soilborne pathogen $F$. oxysporum f. sp. cucumerinum on the subsequent colonization of $F$. oxysporum $\mathrm{f}$. sp. cucumerinum (Fig. 1 , experiment B) was investigated in a split-root system without actual contact between the two inoculants. Suspensions of SQR9, F. oxysporum f. sp. cucumerinum, the PGPR strain Pseudomonas brassicacearum $\mathrm{J} 12$, or the non-PGPR strain Bacillus subtilis 168 were injected to the left side of the split roots (no inoculation was termed $\mathrm{CK}$ ), and then, the colonization of $F$. oxysporum f. sp. cucumerinum on the right side was measured. In this way, we could focus on the systemic response of cucumber to inoculation that would, in turn, affect the colonization of SQR9 or F. oxysporum f. sp. cucumerinum without considering the disturbance of direct microbe-microbe interaction. Preinoculation of either the PGPR strain SQR9 or the plant soil-borne pathogen $F$. oxysporum f. sp. cucumerinum in one side of the split-root system inhibited the subsequent colonization of $F$. oxysporum f. sp. cucumerinum on the other side (Fig. 2A). When cucumber roots were preinoculated by $P$. brassicacearum J12 (a PGPR control from a different genus of SQR9) or B. subtilis 168 (a non-PGPR control from the same genus of SQR9), subsequent $F$. oxysporum f. sp. cucumerinum colonization was also inhibited (Fig. 2A). In a separate experiment, the influence of previous root colonization by a different microbe on the subsequent colonization of SQR9 (Fig. 1, experiment A) was investigated. Infection with $F$. oxysporum f. sp. cucumerinum resulted in enhanced root colonization of
SQR9, as reported previously (Liu et al. 2014). Interestingly, preinoculation of SQR9 also stimulated its own subsequent root colonization (Fig. 2B), while J12 and 168 had no effect on the subsequent root colonization of SQR9 (Fig. 2B).
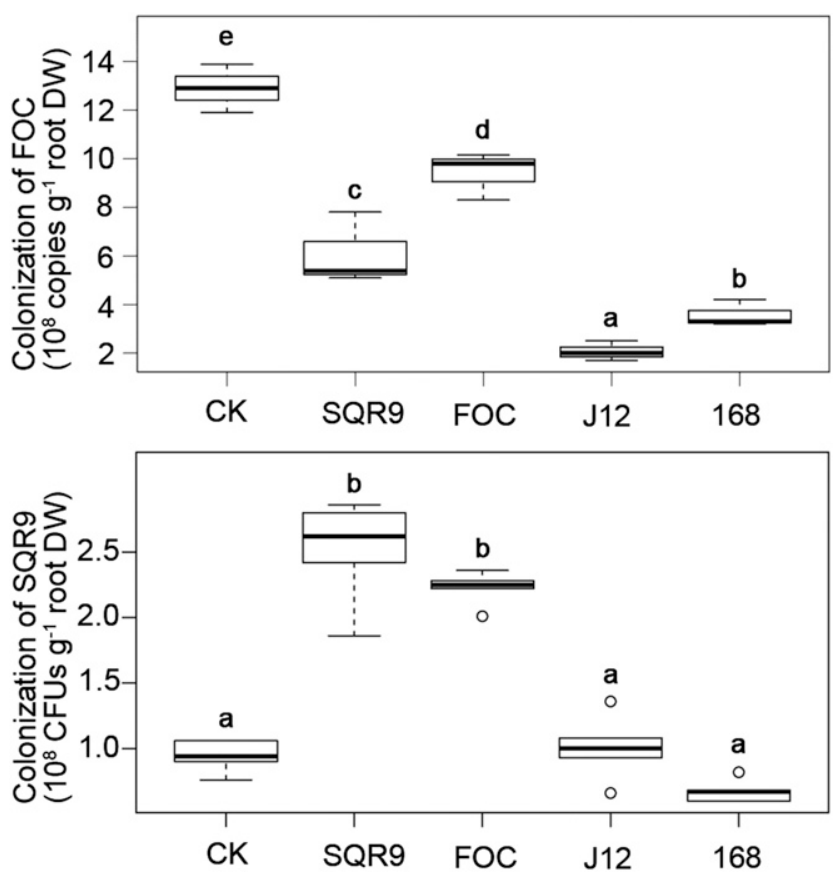

Fig. 2. Colonizations in a split-root system. Top, Colonization of Fusarium oxysporum f. sp. cucumerinum (FOC) was shown in copy number normalized with root dry weight. Effect of preinoculation of Bacillus amyloliquefaciens SQR9, Fusarium oxysporum f. sp. cucumerinum, Pseudomonas brassicacearum $\mathrm{J} 12$, or $B$. subtilis 168 to one side of the split-root system on colonization of $F$. oxysporum f. sp. cucumerinum in another side was measured, using the real time polymerase chain reaction method. Treatment without inoculation in the left side was termed CK. Bottom, Colonization of SQR9 was shown by CFU count on Luria Bertani agar plate. Effect of preinoculation of SQR9, F. oxysporum f. sp. cucumerinum, J12, or B. subtilis 168 to one side of the splitroot system on colonization of SQR9 in another side was measured using plate counting. Treatment without inoculation in the left side was termed CK. For each column, error bars indicate the standard deviations based on three different replicated experimental values. Each replicate was measured three times. Different letters above the bars indicate significant differences $(P<0.05)$.

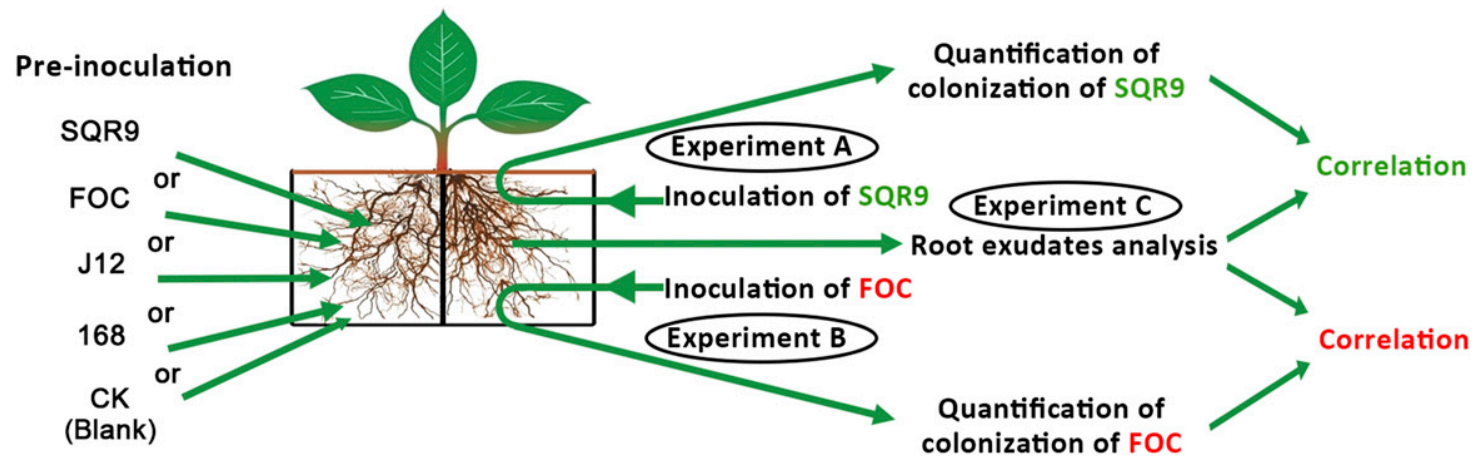

Fig. 1. Experimental design. Experiments were performed in a split-root system. Experiment A, Bacillus amyloliquefaciens SQR9, Fusarium oxysporum f. sp. cucumerinum (FOC), Pseudomonas brassicacearum J12, or B. subtilis 168 was preinoculated into the left chamber, respectively. No inoculation was termed CK. After a 48-h precolonization, suspension of SQR9 was added into the right chamber. After $48 \mathrm{~h}$ more, the colonization of the subsequently inoculated SQR9 was evaluated. Three replicates were included for each treatment. Experiment B, B. amyloliquefaciens SQR9, F. oxysporum f. sp. cucumerinum, P. brassicacearum J12, or B. subtilis 168 was preinoculated into the left chamber, respectively. After a 48-h precolonization, suspension of $F$. oxysporum $\mathrm{f}$. sp. cucumerinum conidia was added into the right chamber. After $48 \mathrm{~h}$ more, the colonization of the subsequently inoculated $F$. oxysporum $\mathrm{f}$. sp. cucumerinum was evaluated. Three replicates were included for each treatment. Experiment $\mathrm{C}$, In a separate experiment, B. amyloliquefaciens SQR9, F. oxysporum $\mathrm{f}$. sp. cucumerinum, P. brassicacearum J12, or B. subtilis 168 was preinoculated into the left chamber, respectively. After a 48 -h precolonization, the medium in the right chamber was replaced by water. The root exudates were collected and performed by gas chromatography-mass spectrometry analysis. Three replicates were included for each treatment. Last, correlation analyses between colonizations (experiments A and B) and root exudates (experiment C) were performed. 


\section{Plant resistance.}

Systemic plant resistance was considered as a factor that affected colonization. Using real time polymerase chain reaction (PCR), transcription of several plant genes involved in systemic resistance were measured for noninoculated root and the leaf. Jasmonic acid (JA), salicylic acid (SA), and ethylene (ET) are known as important hormones that lead to systemic resistance. Allene oxide synthase (AOS) is vital for the biosynthesis of JA (Kazan and Manners 2008). ET response factor 1 (ERF1) is a downstream component of the ET signaling pathway (Lorenzo et al. 2003). The nonexpressor of pathogenesis-related (PR) genes 1 (NPR1), whose expression is induced by SA, is a key regulator of plant systemic resistance (Ding et al. 2016). Phytoalexin deficient 4 (PAD4) is important for the accumulation of SA in plant tissue. A significant increase in transcription of ERF1 and PAD4 upon inoculation of the pathogen F. oxysporum f. sp. cucumerinum was observed in both root and leaf (Fig. 3). As PR3 and PR4 were both under the regulation of the JA and ET pathway, the change was quite similar both in leaf and root. Differing from expectations, inoculation of SQR9, J12, and 168 caused a sharp decrease of the PR proteins. Inoculation of $F$. oxysporum f. sp. cucumerinum did not show any effect on the transcription of these five PR proteins in root (Fig. 3). The phenomenon in leaf and root is similar, indicating a systemic effect has been activated.

\section{Composition of root exudates.}

Because root exudates are recognized as the source of crosstalk signals in the rhizosphere and mediate interactions between the PGPR, roots, and soil-borne pathogens (Badri and Vivanco 2009), we wanted to identify the signals within the root exudates that mediate the tripartite interaction of PGPR, plant roots, and soil-borne pathogens that influenced their root colonization. To this end, root exudates from the cucumber inoculated with SQR9, J12, 168, and F. oxysporum f. sp. cucumerinum were collected and analyzed. The inoculants were in the left side without actual contact to the collection side. In total, 174 compounds were identified and all of them existed in the five treatments of CK (no inoculation), SQR9 (inoculated with SQR9 in one root side), F. oxysporum f. sp. cucumerinum (inoculated with $F$. oxysporum f. sp. cucumerinum in one root side), J12 (inoculated with $\mathrm{J} 12$ in one root side), and 168 (inoculated with 168 in one root side). Treatment without inoculation in the left side was termed CK (Fig. 1, experiment C). However, the abundance of each particular compound varied among the different treatments. Principal component analysis (PCA) showed that principal components 1 and 2 accounted for 76.3 and $17.9 \%$ of the effects, respectively (Fig. 4A). Five treatments were clearly distinct and the CK group differed greatly from the other four treatments, significant difference between the treatments was observed based on ADONIS $(P=0.001)$ (Fig. 4A). In addition, similar change trends within root exudates were observed with SQR9 and J12 in PCA (Fig. 4A) as were also observed with F. oxysporum $\mathrm{f}$. sp. cucumerinum and 168. Among the 174 compounds, 109 were classified and categorized into nine groups based on specific structure. In total, eight amines, 17 amino acids, 20 sugars, six sugar acids, 13 sugar alcohols, nine fatty acids, 18 organic acids, and 18 compounds grouped as 'others' were identified (Table 1). Significances of the changes were calculated and are shown in Supplementary Table S2. Compared with control cucumber root exudates, preinoculation of SQR9 significantly decreased 52 compounds and increased only 13 compounds in the root exudates (Table 1). Preinoculation of $F$. oxysporum f. sp. cucumerinum significantly decreased 31 compounds and increased five compounds in the root exudates (Table 1). Preinoculation of another PGPR strain, J12, significantly decreased 37 compounds and increased 19 compounds in the root exudates (Table 1). Preinoculation of lab-domesticated B. subtilis 168 also significantly altered the cucumber root exudates, with 13 compounds being decreased and 34 compounds being increased (Table 1).

The composition of the different groups (amines, amino acids, sugars, sugar acids, sugar alcohols, fatty acids, organic acids, and others) is shown in Figure 4B. The varied regulation of the exudate compounds is shown in a heatmap (Fig. 4C) that also shows the changes resulting from the F. oxysporum f. sp. cucumerinum and 168 treatments were similar while those resulting from the SQR9 and J12 treatments were similar.

Correlation analysis of the abundance of the compounds in the root exudates and root colonization of SQR9 and $F$. oxysporum f. sp. cucumerinum.

To screen the potential signals in root exudates that correlate to root colonization of SQR9 and $F$. oxysporum f. sp. cucumerinum, Spearman correlation coefficients between root colonization of SQR9 or F. oxysporum f. sp. cucumerinum and the abundance of the compounds in the root exudates were calculated (Fig. 5A; Table 2; Supplementary Table S3). Root colonization of $F$. oxysporum f. sp. cucumerinum positively correlated with a range of sugars, fatty acids, and sugar
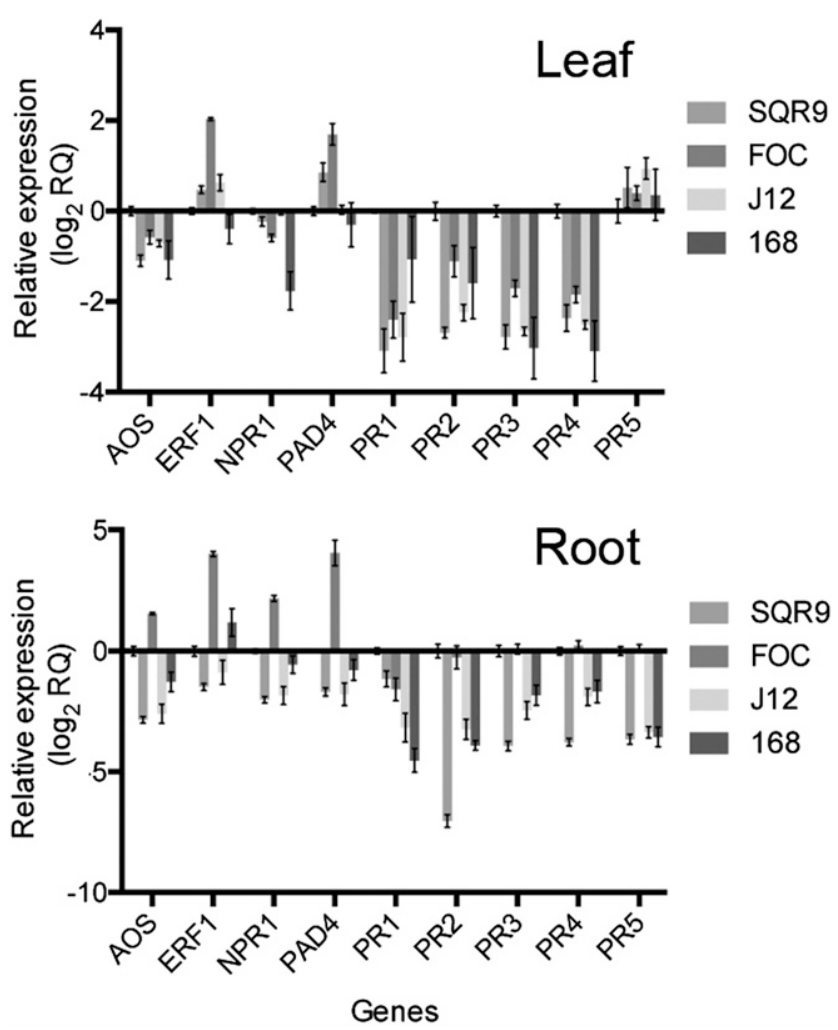

Fig. 3. Transcription of genes involved in plant resistance upon inoculations. AOS, allene oxide synthase (Csa2M360780.1); ERF1, ethylene response factor1 (Csa3M135120.1); NPR1, nonexpressor of pathogenesisrelated (PR) genes 1 (Csa4M063470.1); PAD4, phytoalexin-deficient 4 (Csa4M496760.1); PR1, PR protein 1 (Csa003482); PR2, PR protein 2 (Csa1M660200.1); PR3, PR protein 3 (Csa6M507520.1); PR4, pathogenesis-related PR protein 4 (Csa6M507520.1); PR5, PR protein 5 (Csa2M361370.1). All genes were normalized using ACT1 (actin) as reference. 
alcohols. Root colonization of SQR9 was negatively correlated with sugar alcohols, fatty acids, and sugars. Based on further calculations of the $P$ values of the correlations, the chemicals with significant correlation to colonization were determined (Table 2). The correlation coefficients of raffinose, lyxitol, shikimate, glucose, and pentitol compared with root colonization of $F$. oxysporum f. sp. cucumerinum and sorbitol, maltitol, and tryptophan to that of SQR9 were $0.87,0.80$,
$-0.69,-0.66,-0.66,-0.71,-0.63$, and 0.56 , respectively (Table 2). Pentitol, glucose, and shikimate were negatively correlated with $F$. oxysporum f. sp. cucumerinum colonization, while lyxitol and raffinose were positively correlated with $F$. oxysporum f. sp. cucumerinum colonization (Table 2). Maltitol and sorbitol were negatively correlated with root colonization of SQR9, with $P$ values lower than 0.01 , while tryptophan was positively correlated with root
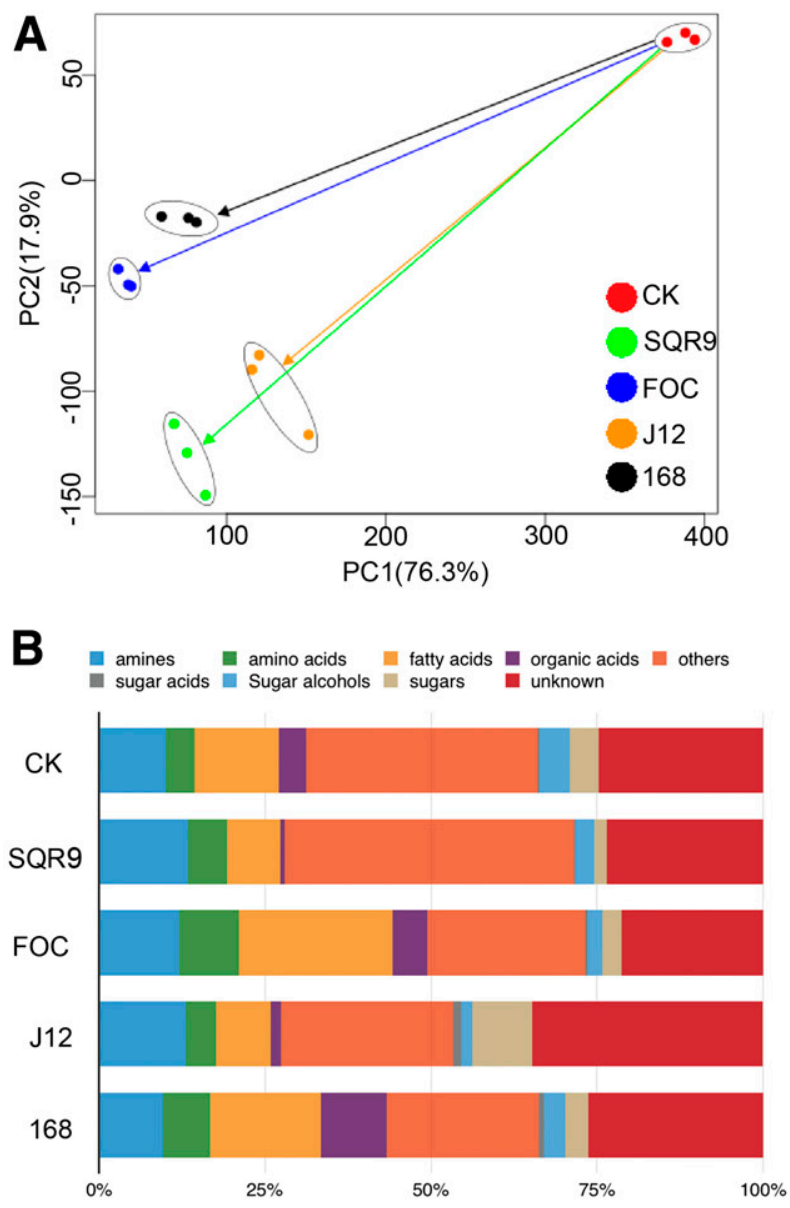



Fig. 4. Gas chromatography-mass spectrometry analysis of root exudates. Regular root exudates from cucumber without preinoculation were termed CK A, Principal component analysis of root exudates in each treatment. An individual point indicates one replicate of the treatment. Prinicipal component 1 contributes $76.3 \%$ and principal component 2 contributes $17.9 \%$ of the factor. Three replicates for each treatment were included. B, Composition of root exudates in group scale. The compounds detected were classified into nine groups: amines, amino acids, fatty acids, organic acids, sugar acids, sugar alcohols, sugars, others, and unknown. For each group, the percentage of the sum of peak heights to the total peak heights is shown. C, Relative content of compound. Relative content to CK (no inoculation) was calculated by using peak height of CK as a denominator. $\log _{10}$ (relative content to CK) was mapped. A blue band indicates a compound in treatment was increased compared with that in CK, while a red band indicates a decrease. The saturation of the color indicates the variation range.

Table 1. Variation of compounds in root exudates ${ }^{\mathrm{a}}$

\begin{tabular}{|c|c|c|c|c|c|c|c|c|c|}
\hline \multirow[b]{2}{*}{ Classifications } & \multirow[b]{2}{*}{ CK } & \multicolumn{2}{|c|}{ SQR9 } & \multicolumn{2}{|c|}{$\begin{array}{l}\text { F. oxysporum f. sp. } \\
\text { cucumerinum }\end{array}$} & \multicolumn{2}{|c|}{ J12 } & \multicolumn{2}{|c|}{168} \\
\hline & & Up & Down & Up & Down & Up & Down & Up & Down \\
\hline Amines & 8 & 2 & 2 & 0 & 4 & 3 & 1 & 1 & 0 \\
\hline Amino acids & 17 & 5 & 6 & 3 & 2 & 5 & 4 & 8 & 0 \\
\hline Sugars & 20 & 2 & 11 & 1 & 8 & 4 & 7 & 2 & 7 \\
\hline Sugar acids & 6 & 0 & 4 & 0 & 2 & 3 & 0 & 4 & 0 \\
\hline Sugar alcohols & 13 & 0 & 7 & 0 & 7 & 2 & 9 & 2 & 4 \\
\hline Fatty acids & 9 & 0 & 6 & 1 & 3 & 0 & 4 & 2 & 0 \\
\hline Organic acids & 18 & 1 & 9 & 0 & 3 & 1 & 5 & 8 & 1 \\
\hline Others & 18 & 3 & 7 & 0 & 2 & 1 & 7 & 7 & 1 \\
\hline Total & 109 & 13 & 52 & 5 & 31 & 19 & 37 & 34 & 13 \\
\hline
\end{tabular}

\footnotetext{
${ }^{a}$ Numbers indicate the number of increased or decreased chemicals in treatment compared with no treatment (CK).
} 
colonization of SQR9 (Table 2). Based on these calculations, the above compounds were selected as potential signals involved in the regulation of root colonization process of F. oxysporum f. sp. cucumerinum and SQR9 in the interaction with cucumber; the pure chemical forms of these compounds were tested further (Table 2).

\section{Effect of the selected chemicals on root colonization of SQR9 and $F$. oxysporum f. sp. cucumerinum.}

When the potential signals possibly affecting the root colonization of SQR9 or F. oxysporum $\mathrm{f}$. sp. cucumerinum had been identified based on the correlation analysis, they were further tested, using pure chemical compounds for their effects on SQR9 or F. oxysporum f. sp. cucumerinum root colonization. The results showed that colonization of $F$. oxysporum f. sp. cucumerinum on cucumber root decreased along with increasing content of pentitol, glucose, and shikimate, which negatively correlated with root colonization of $F$. oxysporum $\mathrm{f}$. sp. cucumerinum (Fig. 5B). Lyxitol and raffinose, which show positive correlation with root colonization of $F$. oxysporum $\mathrm{f}$. sp. cucumerinum, strongly increased $F$. oxysporum f. sp. cucumerinum colonization (Fig. 5B). Figure 5 matches the trends in the effects of the chemicals on $F$. oxysporum f. sp. cucumerinum colonization with the correlation results showing that pentitol, glucose, and shikimate were negatively correlated with $F$. oxysporum f. sp. cucumerinum colonization while lyxitol and raffinose were positively correlated. However, none of these chemicals showed positive effect on F. oxysporum f. sp. cucumerinum growth (Supplementary Fig. S1).

Tryptophan and maltitol enhanced SQR9 root colonization at concentrations ranging from 1 to $100 \mathrm{mg}$ per liter, and both of the chemicals enhanced biofilm formation of SQR9 (Supplementary Fig. S2), while neither of them acted as a chemoattractant (Supplementary Fig. S3). The effect of sorbitol on SQR9 root colonization was dose-dependent (Fig. 5B); SQR9 colonization increased along with increasing sorbitol concentrations, but sorbitol did not affect the biofilm or chemotaxis of SQR9. The pure chemical

testing results of maltitol and sorbitol on root colonization of SQR9 were inconsistent with the correlation analysis, which indicated that the presence of maltitol and sorbitol in root exudates were both negatively correlated with SQR9 root colonization.

\section{Relative content of the correlated compounds in root exudates.}

After testing the effect of chemicals on root colonization of SQR9 or F. oxysporum f. sp. cucumerinum, the relative content of the eight compounds correlated to the colonization of SQR9 or F. oxysporum f. sp. cucumerinum in root exudates were listed to confirm their significance in interaction with cucumber (Fig. 6). Concentrations of pentitol, lyxitol, shikimate, and maltitol in root exudates following the SQR9, F. oxysporum f. sp. cucumerinum, and CK treatments did not show significant differences. Glucose and sorbitol concentrations were inconsistent between the correlation analysis, colonization, and the relative contents in root exudates following the treatments. So pentitol, lyxitol, shikimate, maltitol, glucose, and sorbitol could not be considered

Table 2. Correlation between root-secreted compounds and colonization of SQR9 and Fusarium oxysporum f. sp. cucumerinum

\begin{tabular}{lcc}
\hline Compound & Correlation & P value \\
\hline Correlation to F. oxysporum f. sp. cucumerinum & \\
Raffinose & $0.87 * *$ & $2.32 \mathrm{E}-05$ \\
Lyxitol & $0.80^{* *}$ & $3.07 \mathrm{E}-04$ \\
Shikimate & $-0.69^{* *}$ & $4.77 \mathrm{E}-03$ \\
Glucose & $-0.66^{* *}$ & $6.91 \mathrm{E}-03$ \\
Pentitol & $-0.66^{* *}$ & $7.12 \mathrm{E}-03$ \\
Correlation to SQR9 & $-0.71^{* *}$ & $3.20 \mathrm{E}-03$ \\
Sorbitol & $-0.63^{* *}$ & $9.98 \mathrm{E}-03$ \\
Maltitol & $0.56^{*}$ & $2.97 \mathrm{E}-02$ \\
Tryptophan & & \\
\hline a & Asterisks indicate that the mean value is significantly different from that \\
of the wild type at the same conditions. One asterisk $(*)$ indicates $P<0.05$ \\
and two asterisks $(* *) P<0.01$.
\end{tabular}
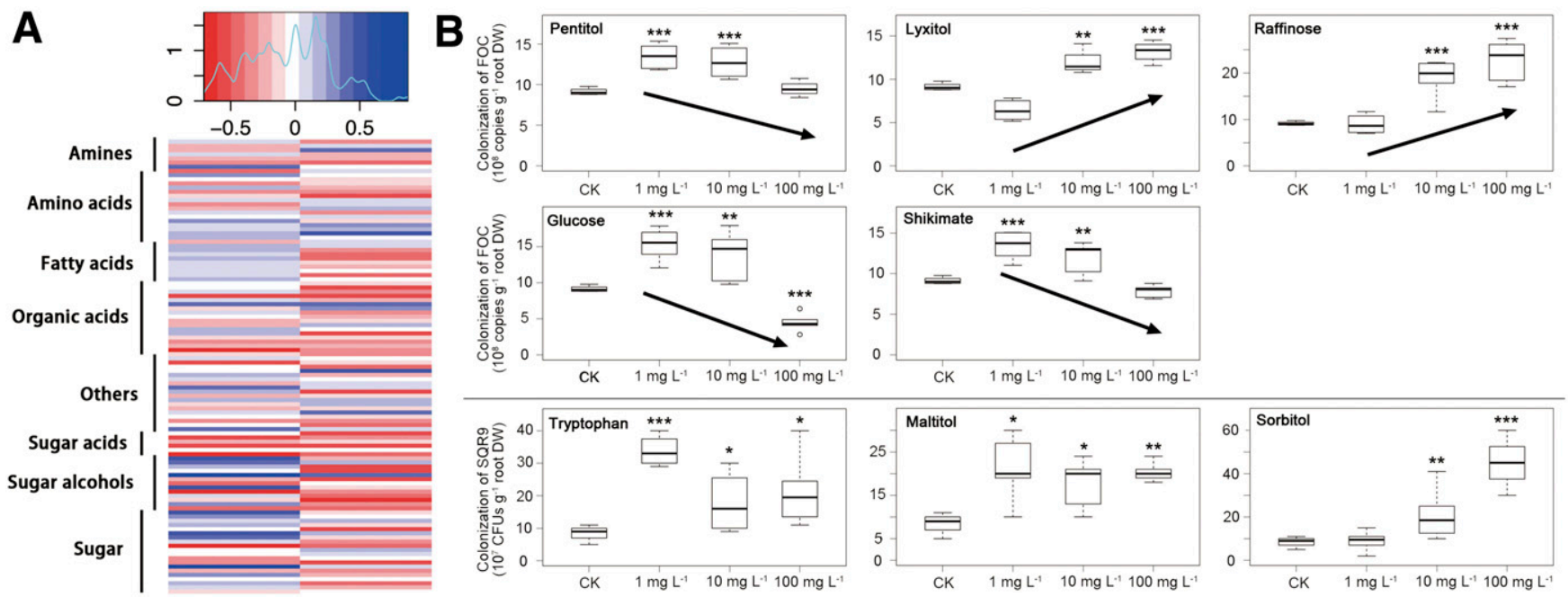

Fig. 5. Correlation between root exudates and colonizations. A, Each compound was correlated with the colonization of SQR9 and Fusarium oxysporum f. sp. cucumerinum. For each operation, 15 values were included. A blue band indicates a high correlation coefficient with relative colonization. A red band indicates a low correlation coefficient. The saturation of the color indicates the variation range. B, Colonization of SQR9 and F. oxysporum f. sp. cucumerinum correlated with the exogenous applied chemical. Colonizations of $F$. oxysporum $\mathrm{f}$. sp. cucumerinum with negatively correlated compounds pentitol, glucose, and shikimate and positively correlated compounds lyxitol and raffinose were quantified using real time polymerase chain reaction. Colonizations of SQR 9 with negatively correlated compounds maltitol and sorbitol and positively correlated compound tryptophan were quantified using plate counting. The arrow above the chart indicates the changing trends along with the concentration of exogenous chemicals. Error bars indicate the standard deviations based on five different replicated experimental values. 
as signals that regulate colonization of SQR9 or $F$. oxysporum f. sp. cucumerinum in the SQR9, cucumber, and F. oxysporum f. sp. cucumerinum interaction.

\section{DISCUSSION}

Efficient root colonization of rhizosphere microbes is known to be crucial for their ability to compete as well as for other necessary functions (Lugtenberg and Kamilova 2009). Plant-microbe interactions in the rhizosphere are complex and influenced by many environmental factors. This study aimed to screen root exudates for signaling compounds, which could mediate the tripartite interaction of Bacillus amyloliquefaciens SQR9, cucumber roots, and soilborne pathogen $F$. oxysporum $\mathrm{f}$. sp. cucumerinum in the rhizosphere. This study confirmed a previous report showing that infection or preinoculation of $F$. oxysporum f. sp. cucumerinum causes an enhancement in the subsequent root colonization of PGPR strain SQR9 (Liu et al. 2014). It is thought that pathogen infection is recognized by plants and, thus, signal transduction leads to changes within the composition of root exudates, which is recognized as the source of communication between plants and other organisms in the rhizosphere (Bais et al. 2006). The concentrations of some compounds in the root exudates were increased, possibly as signals, and attract more SQR9 to fight against $F$. oxysporum f. sp. cucumerinum. Likewise, in this study, preinoculation with SQR9 enhanced its own subsequent root colonization. There are also reports showing the effect in fungi (Catford et al. 2003; Vierheilig 2004). Nevertheless, our understanding of the mechanisms behind this phenomenon is extremely poor. When other strains were tested in our system, another PGPR strain, J12, which was isolated from the rhizosphere of tomato plants (Zhou et al. 2012), did not cause enhanced SQR9 colonization. It has been suggested that long-term coevolution of plant hosts with their associated rhizosphere microbes might explain some of these specific interactions, as we reported previously (Liu et al. 2014; Zhang et al. 2014).

F. oxysporum f. sp. cucumerinum colonization on cucumber roots decreased upon pre-exposure to PGPR strains (SQR9 and $\mathrm{J} 12$ ) and $F$. oxysporum f. sp. cucumerinum itself in this study. Martinuz et al. (2012) reported similar results, in that inoculation of either beneficial bacteria or fungal pathogens in a split-root system led to a reduction in subsequent pathogen colonization. Surprisingly, preinoculation of the labdomesticated non-PGPR strain 168 also inhibited F. oxysporum f. sp. cucumerinum colonization. Previous study showed that both $B$. amyloliquefaciens SQR9 and $P$. brassicacearum $\mathrm{J} 12$ antagonize the fungal pathogen $F$. oxysporum $\mathrm{f}$. sp. cucumerinum by synthesizing antibiotics (Xu et al. 2013; Zhou et al. 2012). It is generally known that a range of secondary metabolites secreted by PGPR, like surfactin and fengycin, are potentially involved in inducing systemic resistance to pathogens (Lee et al. 2012; Ongena et al. 2007; Yi et al. 2013). However, the inoculation of the PGPR strains in this study (SQR9 and J12) caused a sharp decrease of transcription of PR proteins in cucumber (Fig. 3). Moreover, the result did not show activation of the PR proteins (Fig. 3) upon inoculation of F. oxysporum f. sp. cucumerinum, although a significant increase of the expression of ERF1 and PAD4 was observed. This is quite different from the phenomenon observed in the model plant (Cartieaux et al. 2008; Tsuda et al. 2008a and b). More research on cucumber resistance would give an insight to the phenomenon.

It is known that root exudates play a vital role in regulating colonization of rhizosphere microbes (Badri and Vivanco 2009; Bais et al. 2008, 2006). To screen for the compounds involved in the regulation of SQR9 and F. oxysporum $\mathrm{f}$. sp. cucumerinum colonization, correlation analysis was performed between root colonization ability and the quantities of certain compounds in
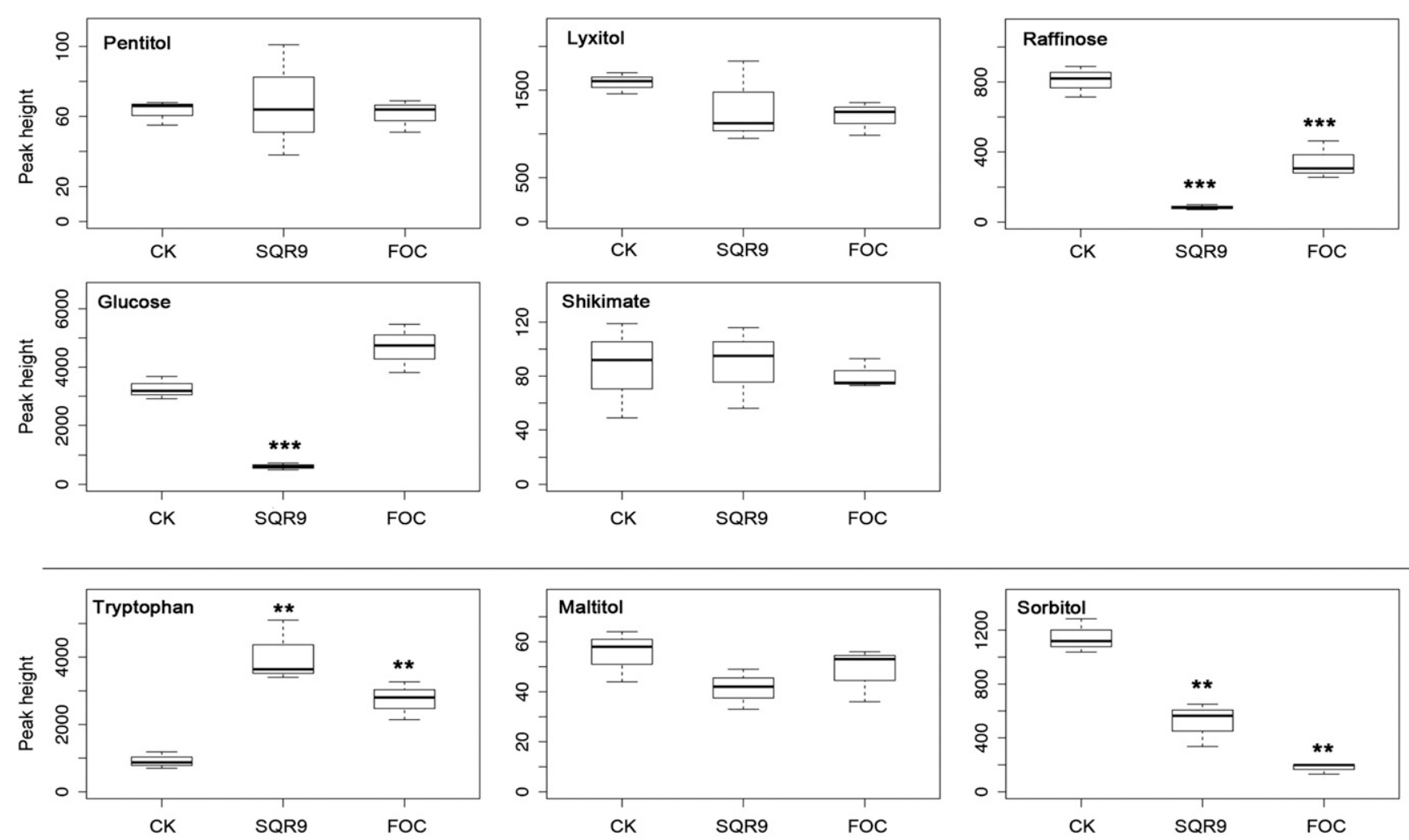

Fig. 6. Relative contents of chemicals in root exudates. Peak height of the chemicals with correlations to colonization were shown. The peak heights in Fusarium oxysporum f. sp. cucumerinum (FOC), SQR9, and CK (no inoculation) treatments were shown. Error bars indicate the standard deviations based on three different replicated experimental values. 
the root exudates to identify candidate signaling compounds. Eight compounds were identified and tested further, among which four were sugar alcohols and two were sugars.

Pentitol, lyxitol, shikimate, and maltitol were not considered to be signals that contributed to root colonization in our experiments because their levels in root exudates following treatment with CK, SQR9, or F. oxysporum f. sp. cucumerinum were not significantly different (Fig. 6). Glucose and sorbitol had inconsistent effects on root colonization, as shown in the root exudates and by correlation analysis (Table 2; Figs. 5 and 6). These compounds did not contribute to any changes in root colonization in the tripartite interactions of SQR9, F. oxysporum f. sp. cucumerinum, and the cucumber roots.

Raffinose, however, had a positive effect on colonization of F. oxysporum f. sp. cucumerinum (Fig. 5B), as shown by correlation analysis (Table 2). The quantity of raffinose in the root exudates decreased sharply upon preinoculation of $F$. oxysporum $\mathrm{f}$. sp. cucumerinum and SQR9 (Fig. 6). This analysis indicates that raffinose positively regulates $F$. oxysporum f. sp. cucumerinum colonization. Exogenous raffinose has been reported to be crucial for rhizosphere competition and protection of plants from oxidative damage (Fang and St. Leger 2010; Nishizawa et al. 2008). Moreover, study on rhizosphere fungi showed that mutations within the raffinose transporter led to poor growth in root exudates and greatly reduced rhizosphere competition (Fang and St. Leger 2010; Nishizawa et al. 2008). However, the function of raffinose in root exudates upon pathogen colonization has not been reported. Conversely, tryptophan, which showed a positive relationship with SQR9 colonization in both the correlation analysis (Table 2) and pure chemical testing experiments (Fig. 5B), should have played a vital role in promoting SQR9 colonization. It has also been reported that increased levels of tryptophan contribute to enhanced rhizosphere colonization of indole 3-acetic acid (IAA)-producing PGPR (Naveed et al. 2015). However, tryptophan is generally known to play a vital role in IAA production in both plants and microbes. Previous study showed the enhanced tryptophan secretion from cucumber colonized by SQR9 contributes to the IAA production of SQR9 (Liu et al. 2016). In this study, we showed the enhanced tryptophan secretion is not only the precursor for IAA production by SQR9 but, also, a signal that stimulates the colonization. It is known that the metabolism of tryptophan in plants is integrated with the production of the systemic resistance signals, such as SA synthesis from chorismate (Radwanski and Last 1995; Shah 2003). However, deeper study should be performed in the future to confirm whether systemic resistance contribute to the change of colonization of F. oxysporum f. sp. cucumerinum and SQR9 on cucumber root and to understand how plant resistance interacts with root exudation.

In this study, based on the analysis of root exudates using gas chromatography-mass spectrometry (GC-MS), we found that cucumber roots could sense microbial signals emanating from SQR9 and F. oxysporum f. sp. cucumerinum within the rhizosphere and subsequently reduce the secretion of raffinose, creating an environment that is not conducive to $F$. oxysporum f. sp. cucumerinum colonization. Likewise, cucumber that sense SQR9 and F. oxysporum f. sp. cucumerinum and subsequently secret more tryptophan to recruit SQR9 benefited the cucumber itself or prevented infection by $F$. oxysporum f. sp. cucumerinum (Fig. 7). Nevertheless, in vivo detection of the exact concentration of these compounds within the rhizosphere would give more information that could enhance our understanding of these complex interactions.

\section{MATERIALS AND METHODS}

Strains and culture conditions.

The cucumber wilt disease fungal pathogen Fusarium oxysporum f. sp. cucumerinum NJAU-2 (F. oxysporum f. sp. cucumerinum NJAU-2) was stored in the Jiangsu Provincial Key Laboratory for Organic Solid Waste Utilization, Nanjing Agricultural University and was maintained on potato dextrose agar plates. For storage, its conidia were mixed with $20 \%$ glycerol and stored in a $-80^{\circ} \mathrm{C}$ freezer. Bacillus amyloliqueficiens SQR9 (China General Microbiology Culture Collection Center, CGMCC accession number 5808), Pseudomonas brassicacearum J12 (National Center for Biotechnology Information accession number JN605747), and Bacillus subtilis 168 (Bacillus Genetic Stock Center) were grown at $37^{\circ} \mathrm{C}$ on Luria-Bertani medium (LB) agar plates.

Cucumber seeds of 'Jinchun 4' were surface-disinfected in a $2 \% \mathrm{NaClO}$ solution for $15 \mathrm{~min}$, were washed thoroughly with distilled water, were planted into axenic tissue culture bottles containing vermiculite, and were allowed to germinate and grow for 4 days in a growth chamber at $23^{\circ} \mathrm{C}$, with a 16 -h light and 8-h dark photoperiod. Seedlings were then transplanted into $50-\mathrm{ml}$ flasks containing $35 \mathrm{ml}$ of sterile liquid $(1 / 4$ sucrosefree Murashige Skoog medium) to submerge the seedling roots in the medium, with one seedling in each flask. Seedlings were cultured in these flasks for 3 weeks. The Murashige Skoog medium was replaced every other day during the growth period. The hydroponic system was gently shaken $(50 \mathrm{rpm})$ for $2 \mathrm{~h}$ each day on a shaker.

The generated seedlings were transferred to the split-root system (six plants in each) and were cultured for 2 more weeks in $1 / 4$ sucrose-free Murashige Skoog medium. Murashige Skoog medium (2 liters) in the system was replaced every

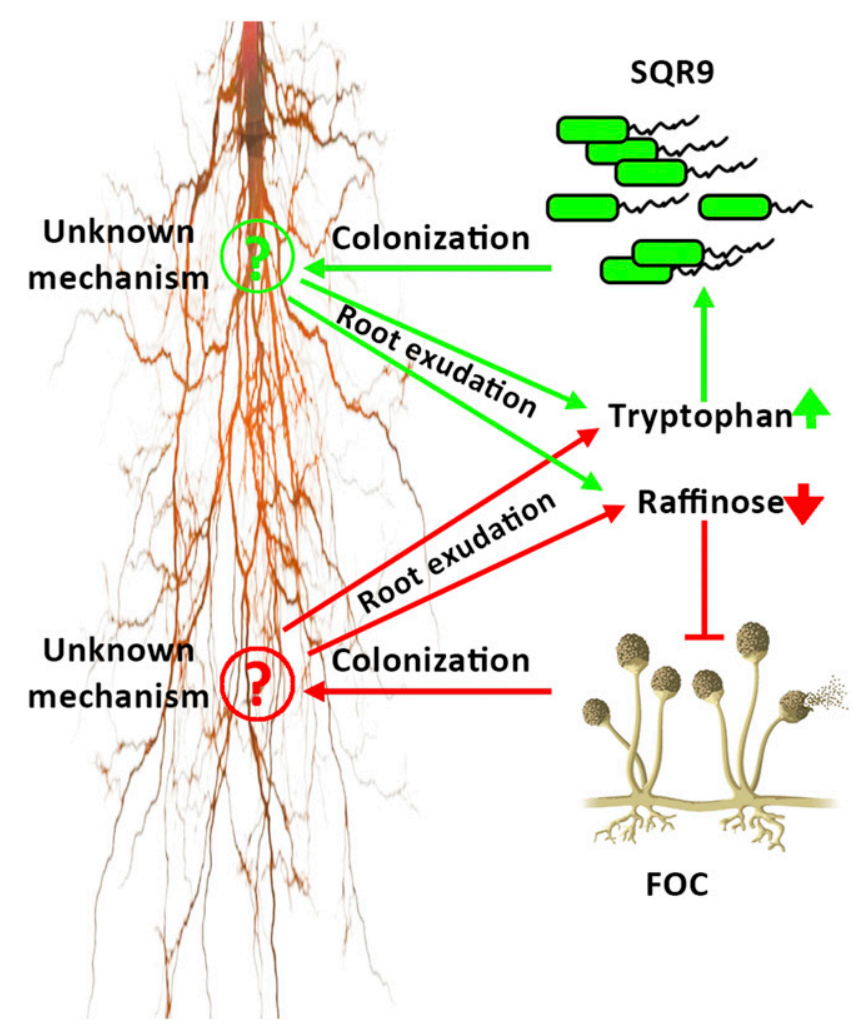

Fig. 7. Interaction mode. Colonization of Bacillus amyloliquefaciens SQR9 could be recognized by cucumber root and consequently enhanced root secretion of tryptophan and reduced that of raffinose. Tryptophan and raffinose are positive factors of Bacillus amyloliquefaciens SQR9 and Fusarium oxysporum, respectively. Therefore, colonization of SQR9 was increased along with the increased tryptophan. In contrast, colonization of F. oxysporum f. sp. cucumerinum was decreased. In another case, colonization of $F$. oxysporum enhanced root secretion of tryptophan and reduced that of raffinose. As a result, colonization of SQR9 increased, and colonization of F. oxysporum f. sp. cucumerinum decreased. 
2 days for ventilation and nutrient addition. All the containers and media were sterilized prior to use. Before inoculation, each device was checked for sterility by taking a $100 \mu \mathrm{l}$ aliquot and spreading it on solid LB plates; contaminated systems were discarded.

\section{Colonization assay.}

F. oxysporum f. sp. cucumerinum conidia suspensions were prepared as described previously (Cao et al. 2011). Suspensions of SQR9, J12, and 168 were prepared in LB medium and were suspended in $1 / 4$ sucrose-free Murashige Skoog medium at an optical density at $600 \mathrm{~nm}$ of 1.0 . Suspensions of $F$. oxysporum f. sp. cucumerinum conidia, SQR9, J12, and 168 were separately inoculated to the left side of the split-root system and were mixed with the medium (Fig. 1). The split-root system was cultured for two more days to allow for pre-exposure to the inoculants and the right side was checked for contamination and, then, $F$. oxysporum f. sp. cucumerinum conidia $\left(1 \times 10^{6}\right.$ spores $\left.\mathrm{ml}^{-1}\right)$ or SQR9 cells $(5 \times$

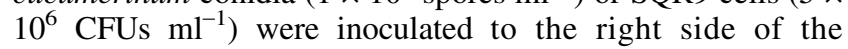
chamber of the split-root system to evaluate the effects of different inoculants on the subsequent root colonization of F. oxysporum f. sp. cucumerinum and SQR9 (Fig. 1A and B). The systems were further cultured for two more days before evaluation. Three replicates (six plants for each) were included for each treatment.

\section{Quantification of colonization.}

For quantitative analysis of SQR9 root colonization, roots in the right side chamber were cut and were briefly washed with sterile double-distilled water. Next, mixed root was weighed and ground with a mortar in sterilized distilled water. The suspensions were then diluted and were plated on LB agar medium. Quantification of root colonization was performed by counting after incubation for $24 \mathrm{~h}$ at $37^{\circ} \mathrm{C}$. The results were normalized to root dry weight.

To evaluate the root colonization of $F$. oxysporum $\mathrm{f}$. sp. cucumerinum, real-time quantitative (q)PCR was performed following the method reported previously (Qiu et al. 2014). A specific fragment in the $F$. oxysporum f. sp. cucumerinum genome was amplified by specific primer pair FocF3 (F) 5' -AAACGAGCCCGCTATTTGAG-3' and FocR7 (R) 5' TATTTCCTCCACATTGCCATG-3'. The resulting product was linked to the T-vector to form a plasmid, which was transformed into DH5 $\alpha$ for duplication. The plasmid was extracted and diluted to generate a standard curve. The roots infected by F. oxysporum f. sp. cucumerinum were washed with sterile water and mixed roots were ground with liquid nitrogen and were subjected to DNA extraction, using a Fungal DNA kit (Omega, Biotek e.Z.N.A.). Real-time PCR was performed to evaluate the cycling threshold of the fungal DNA sample and the plasmid. The copy numbers were calculated using the following equation and the results were normalized to the root dry weight.

$$
D N A(\text { copy })=\frac{6.02 \times 10^{23}\left(\text { copies } \mathrm{mol}^{-1}\right) \times \mathrm{DNA}_{\text {amount }}(\mathrm{g})}{\text { DNA length }(\mathrm{bp}) \times 660\left(\mathrm{~g} \mathrm{~mol}^{-1} \mathrm{bp}^{-1}\right)}
$$

\section{Quantification of transcription of plant resistance gene.}

RNA of cucumbers after inoculation of SQR9, F. oxysporum f. sp. cucumerinum, J12, or 168 was extracted using RNeasy plant mini kit (Qiagen). Reverse transcription PCR was performed using a PrimeScript RT reagent kit with gDNA eraser (Takara). Real time qPCR was performed using an ABI 7500 Cycler (Applied Biosystems). The 20- $\mu$ l reaction solution consisted of $10 \mu$ l of SYBR Premix EX Taq $(2 \times$; TaKaRa), $0.4 \mu \mathrm{l}$ of each primer $(10 \mu \mathrm{M}), 0.4 \mu \mathrm{l}$ of ROX reference dye II (50x), $2 \mu \mathrm{l}$ of template DNA, and $6.8 \mu \mathrm{l}$ of double distilled $\mathrm{H}_{2} \mathrm{O}$. The thermal cycling conditions were set to the following: $30 \mathrm{~s}$ at $95^{\circ} \mathrm{C}$ for initial denaturation, followed by 40 cycles of $5 \mathrm{~s}$ at $95^{\circ} \mathrm{C}$ and $34 \mathrm{~s}$ at $60^{\circ} \mathrm{C}$. The target genes in cucumber plants that encode AOS (Csa2M360780.1), ERF1 (Csa3M135120.1), NPR1 (Csa4M063470.1), PAD4 (Csa4M496760.1), PR1 (Csa003482), PR2 (Csa1M660200.1), PR3 (Csa6M507520.1), PR4 (Csa6M507520.1), and PR5 (Csa2M361370.1) were quantified using an ACT1 gene as an internal reference (Huang et al. 2009); the primers are shown in Supplementary Table S1.

\section{Root exudates collection.}

Suspensions of $F$. oxysporum f. sp. cucumerinum conidia, SQR9, J12, and 168 were separately inoculated to the left side of the split-root system and were mixed with the medium (Fig. 1). The split-root system was cultured for two more days to allow for pre-exposure to the inoculants and the right side was checked for contamination. Medium in the right side of the chamber (not inoculated) was then discarded and replaced with sterile double-distilled water. Over the next four days, the systems were cultured at $23^{\circ} \mathrm{C}$ with a 16 -h light and 8 -h dark photoperiod and the water was collected and replaced every day for root exudates collection (Fig. 1, experiment C). The treatments were repeated three times, including six plants for each replicate. Regular root exudates of cucumber without inoculation were collected as CK. A total of 4 liters of root exudates containing water were collected for each replicate from six plants. Root exudate solutions were filtered through a $0.45-\mu \mathrm{m}$ membrane (Millipore) and were lyophilized. The sterility of the exudates after filtration was tested by plating $100 \mu \mathrm{l}$ of each of the root exudates on a LB plate and incubating at $30^{\circ} \mathrm{C}$, to check the contamination. The root parts immersed under the water were cut and dried for weighing.

\section{Root exudates analysis.}

GC-MS was performed to analyze the root exudates. Freezedried root exudates were shipped to the Genome Center Core Services at the University of California-Davis for analysis. Briefly, root exudates were turned to powder by derivatization (Sana et al. 2010). The samples were run through an Agilent 6890 gas chromatograph containing a $30 \mathrm{~m}$ long, $0.25 \mathrm{~mm}$ i.d. rtx5Sil-MS column with an additional 10-m integrated guard column. The running program was controlled by Leco Chroma TOF software, version 2.32. Resulting files containing absolute spectra intensities were exported to a data server to be processed by a filtering algorithm implemented in the metabolomics BinBase database (Fiehn et al. 2005). Peak height was reported for quantification purposes. The metabolites were correlated to BinBase identifier numbers using mass spectra and retention indices as the identification criteria. Additional confidence criteria were applied, using mass spectral metadata through a combination of unique ions, apex ions, peak purity, and signal/noise ratios. All database entries in BinBase were matched against the Fiehn mass spectral library (University of California Davis). For each treatment, $\log _{10}$ (T/CK) was calculated for evaluation of the range of up- or downregulation. PCA and ADONIS analysis were performed using the R program with the "vegan" library.

\section{Correlation analysis.}

The Spearman correlations between $\mathrm{SQR} 9$ or $F$. oxysporum f. sp. cucumerinum root colonization and the compounds in the root exudates were calculated using the R program. The copy numbers of $F$. oxysporum $\mathrm{f}$. sp. cucumerinum in colonization results and plate counting of SQR9 were normalized to root dry weight. Each of the 15 values (five treatments with three replicates) of transformed colonization results was correlated 
with the relative content of each chemical compound found in the GC-MS data of each treatment. A significant correlation is indicated by a $P$ value of $<0.05$. The compounds with a $P$ value lower than 0.01 were taken to be a potential signal and were further tested by experiments with the selected pure compounds.

\section{Colonization assay with exogenous chemicals.}

The compounds that correlated significantly with root colonization were regarded as potential signals. Standard pure chemicals were purchased for evaluating their effect on root colonization. Cucumber seedlings were allowed to grow for 40 days. Solutions of each pure chemical were prepared with sterile water and were then added to the rhizosphere of the hydroponic system. For each of the chemicals, the final concentrations were 1,10 , and $100 \mathrm{mg}$ per liter, respectively. Suspensions of $F$. oxysporum f. sp. сuсumerinum spores or SQR9 cells were then inoculated to a final concentration of $10^{6}$ spores or $5 \times 10^{6} \mathrm{CFUs}$ per milliliter, respectively. Quantification was performed two days later, using real-time PCR or plate counting as described above.

\section{Statistical analysis.}

Differences among the treatments were calculated and statistically analyzed using analysis of variance and Duncan's multiple range tests $(P<0.05)$ for colonization and comparison of root exudates, respectively. Student's $t$ test was performed for evaluating the significance of root exudates and the effect of chemicals on colonization. The correlation analysis and the calculation of the $P$ values were performed by the R program. SPSS version 22.0 was used for statistical analysis (SPSS Inc.).

\section{ACKNOWLEDGMENTS}

This research was financially supported by the National Natural Science Foundation of China (31330069, 41271271, and 31600088), China Postdoctoral Science Foundation (2016M591297), the National Key Basic Research Program of China (973 program, 2015CB150505), and the National Infrastructure of Microbial Resources (NIRM). R. Zhang and Q. Shen were also supported by the 111 Project (B12009) and the Priority Academic Program Development (PAPD) of Jiangsu Higher Education Institutions.

\section{LITERATURE CITED}

Badri, D. V., and Vivanco, J. M. 2009. Regulation and function of root exudates. Plant Cell Environ. 32:666-681.

Bais, H. P., Broeckling, C. D., and Vivanco, J. M. 2008. Root exudates modulate plant-microbe interactions in the rhizosphere. Pages 241-252 in: Secondary Metabolites in Soil Ecology, Vol. 14. Springer, Berlin.

Bais, H. P., Weir, T. L., Perry, L. G., Gilroy, S., and Vivanco, J. M. 2006. The role of root exudates in rhizosphere interactions with plants and other organisms. Annu. Rev. Plant Biol. 57:233-266.

Cao, Y., Xu, Z., Ling, N., Yuan, Y., Yang, X., Chen, L., Shen, B., and Shen, Q. 2012. Isolation and identification of lipopeptides produced by B. subtilis SQR9 for suppressing Fusarium wilt of cucumber. Sci. Hortic. (Amsterdam) 135:32-39.

Cao, Y., Zhang, Z., Ling, N., Yuan, Y., Zheng, X., Shen, B., and Shen, Q. 2011. Bacillus subtilis SQR9 can control Fusarium wilt in cucumber by colonizing plant roots. Biol. Fertil. Soils 47:495-506.

Cartieaux, F., Contesto, C., Gallou, A., Desbrosses, G., Kopka, J., Taconnat, L., Renou, J.-P., and Touraine, B. 2008. Simultaneous interaction of Arabidopsis thaliana with Bradyrhizobium Sp. strain ORS278 and Pseudomonas syringae pv. tomato DC3000 leads to complex transcriptome changes. Mol. PlantMicrobe Interact. 21:244-259.

Catford, J. G., Staehelin, C., Lerat, S., Piché, Y., and Vierheilig, H. 2003. Suppression of arbuscular mycorrhizal colonization and nodulation in split-root systems of alfalfa after pre-inoculation and treatment with Nod factors. J. Exp. Bot. 54:1481-1487.

Ding, Y., Dommel, M., and Mou, Z. 2016. Abscisic acid promotes proteasome-mediated degradation of the transcription coactivator NPR1 in Arabidopsis thaliana. Plant J. 86:20-34.

Fang, W., and St. Leger, R. J. 2010. Mrt, a gene unique to fungi, encodes an oligosaccharide transporter and facilitates rhizosphere competency in Metarhizium robertsii. Plant Physiol. 154:1549-1557.
Fiehn, O., Wohlgemuth, G., and Sholz, M. 2005. Setup and annotation of metabolomic experiments by integrating biological and mass spectrometric metadata. Pages 224-239 in: Data Integration in the Life Sciences. Vol. 3615. B. Ludäscher and L. Raschid, eds. Springer, Berlin.

Huang, S., Li, R., Zhang, Z., Li, L., Gu, X., Fan, W., Lucas, W. J., Wang, X., Xie, B., Ni, P., Ren, Y., Zhu, H., Li, J., Lin, K., Jin, W., Fei, Z., Li, G. Staub, J., Kilian, A., van der Vossen, E. A., Wu, Y., Guo, J., He, J., Jia, Z., Ren, Y., Tian, G., Lu, Y., Ruan, J., Qian, W., Wang, M., Huang, Q., Li, B., Xuan, Z., Cao, J., Asan, Wu, Z., Zhang, J., Cai, Q., Bai, Y., Zhao, B., Han, Y., Li, Y., Li, X., Wang, S., Shi, Q., Liu, S., Cho, W. K., Kim, J. Y., Xu, Y., Heller-Uszynska, K., Miao, H., Cheng, Z., Zhang, S., Wu, J., Yang, Y., Kang, H., Li, M., Liang, H., Ren, X., Shi, Z., Wen, M., Jian, M., Yang, H., Zhang, G., Yang, Z., Chen, R., Liu, S., Li, J., Ma, L., Liu, H., Zhou, Y., Zhao, J., Fang, X., Li, G., Fang, L., Li, Y., Liu, D., Zheng, H., Zhang, Y., Qin, N., Li, Z., Yang, G., Yang, S., Bolund, L., Kristiansen, K., Zheng, H., Li, S., Zhang, X., Yang, H., Wang, J., Sun, R., Zhang, B., Jiang, S., Wang, J., Du, Y., and Li, S. 2009. The genome of the cucumber, Cucumis sativus L. Nat. Genet. 41:1275-1281.

Kazan, K., and Manners, J. M. 2008. Jasmonate signaling: Toward an integrated view. Plant Physiol. 146:1459-1468.

Lee, B., Farag, M. A., Park, H. B., Kloepper, J. W., Lee, S. H., and Ryu, C.-M. 2012. Induced resistance by a long-chain bacterial volatile: Elicitation of plant systemic defense by a $\mathrm{C} 13$ volatile produced by Paenibacillus polymyxa. PLoS One 7:e48744.

Liu, Y., Chen, L., Zhang, N., Li, Z., Zhang, G., Xu, Y., Shen, Q., and Zhang, R. 2016. Plant-microbe communication enhances auxin biosynthesis by a root-associated bacterium, Bacillus amyloliquefaciens SQR9. Mol Plant-Microbe Interact. 29:324-330.

Liu, Y., Zhang, N., Qiu, M., Feng, H., Vivanco, J. M., Shen, Q., and Zhang, R. 2014. Enhanced rhizosphere colonization of beneficial Bacillus amyloliquefaciens SQR9 by pathogen infection. FEMS Microbiol. Lett. 353:49-56.

Lorenzo, O., Piqueras, R., Sánchez-Serrano, J. J., and Solano, R. 2003. ETHYLENE RESPONSE FACTOR1 integrates signals from ethylene and jasmonate pathways in plant defense. Plant Cell 15:165-178.

Lugtenberg, B., and Kamilova, F. 2009. Plant-growth-promoting rhizobacteria. Annu. Rev. Microbiol. 63:541-556.

Lugtenberg, B. J. J., Kravchenko, L. V., and Simons, M. 1999. Tomato seed and root exudate sugars: Composition, utilization by Pseudomonas biocontrol strains and role in rhizosphere colonization. Environ. Microbiol. 1:439-446.

Martinuz, A., Schouten, A., and Sikora, R. A. 2012. Systemically induced resistance and microbial competitive exclusion: Implications on biological control. Phytopathology 102:260-266.

Naveed, M., Qureshi, M. A., Zahir, Z. a., Hussain, M. B., Sessitsch, A., and Mitter, B. 2015. L-tryptophan-dependent biosynthesis of indole-3-acetic acid (IAA) improves plant growth and colonization of maize by Burkholderia phytofirmans PsJN. Ann. Microbiol. 65: 1381-1389.

Nishizawa, A., Yabuta, Y., and Shigeoka, S. 2008. Galactinol and raffinose constitute a novel function to protect plants from oxidative damage. Plant Physiol. 147:1251-1263.

Ongena, M., Jourdan, E., Adam, A., Paquot, M., Brans, A., Joris, B., Arpigny, J.-L. L., and Thonart, P. 2007. Surfactin and fengycin lipopeptides of Bacillus subtilis as elicitors of induced systemic resistance in plants. Environ. Microbiol. 9:1084-1090.

Qiu, M., Li, S., Zhou, X., Cui, X., Vivanco, J. M., Zhang, N., Shen, Q., and Zhang, R. 2014. De-coupling of root-microbiome associations followed by antagonist inoculation improves rhizosphere soil suppressiveness. Biol. Fertil. Soils 50:217-224.

Qiu, M., Zhang, R., Xue, C., Zhang, S., Li, S., Zhang, N., and Shen, Q. 2012. Application of bio-organic fertilizer can control Fusarium wilt of cucumber plants by regulating microbial community of rhizosphere soil. Biol. Fertil. Soils 48:807-816.

Raaijmakers, J. M., Paulitz, T. C., Steinberg, C., Alabouvette, C., and Moënne-Loccoz, Y. 2009. The rhizosphere: A playground and battlefield for soilborne pathogens and beneficial microorganisms. Plant Soil 321: 341-361.

Radwanski, E. R., and Last, R. L. 1995. Tryptophan biosynthesis and metabolism: Biochemical and molecular genetics. Plant Cell 7:921-934.

Rudrappa, T., Czymmek, K. J., Paré, P. W., and Bais, H. P. 2008. Rootsecreted malic acid recruits beneficial soil bacteria. Plant Physiol. 148: 1547-1556.

Sana, T. R., Fischer, S., Wohlgemuth, G., Katrekar, A., Jung, K.-H., Ronald, P. C., and Fiehn, O. 2010. Metabolomic and transcriptomic analysis of the rice response to the bacterial blight pathogen Xanthomonas oryzae pv. oryzae. Metabolomics 6:451-465. 
Shah, J. 2003. The salicylic acid loop in plant defense. Curr. Opin. Plant Biol. 6:365-371.

Tsuda, K., Glazebrook, J., and Katagiri, F. 2008a. The interplay between MAMP and SA signaling. Plant Signal. Behav. 3:359-361.

Tsuda, K., Sato, M., Glazebrook, J., Cohen, J. D., and Katagiri, F. 2008b. Interplay between MAMP-triggered and SA-mediated defense responses. Plant J. 53:763-775.

Vierheilig, H. 2004. Further root colonization by arbuscular mycorrhizal fungi in already mycorrhizal plants is suppressed after a critical level of root colonization. J. Plant Physiol. 161:339-341.

Wang, B., Li, R., Ruan, Y., Ou, Y., Zhao, Y., and Shen, Q. 2015. Pineapplebanana rotation reduced the amount of Fusarium oxysporum more than maize-banana rotation mainly through modulating fungal communities. Soil Biol. Biochem. 86:77-86.

Xu, Z., Shao, J., Li, B., Yan, X., Shen, Q., and Zhang, R. 2013. Contribution of bacillomycin D in Bacillus amyloliquefaciens SQR9 to antifungal activity and biofilm formation. Appl. Environ. Microbiol. 79:808-815.
Ye, S. F., Yu, J. Q., Peng, Y. H., Zheng, J. H., and Zou, L. Y. 2004. Incidence of Fusarium wilt in Cucumis sativus L. is promoted by cinnamic acid, an autotoxin in root exudates. Plant Soil 263:143-150.

Yi, H.-S., Yang, J. W., and Ryu, C.-M. 2013. ISR meets SAR outside: Additive action of the endophyte Bacillus pumilus INR7 and the chemical inducer, benzothiadiazole, on induced resistance against bacterial spot in field-grown pepper. Front. Plant Sci. 4:122.

Zhang, N., Wang, D., Liu, Y., Li, S., Shen, Q., and Zhang, R. 2014. Effects of different plant root exudates and their organic acid components on chemotaxis, biofilm formation and colonization by beneficial rhizosphere-associated bacterial strains. Plant Soil 374:689-700.

Zhang, S., Raza, W., Yang, X., Hu, J., Huang, Q., Xu, Y., Liu, X., Ran, W., and Shen, Q. 2008. Control of Fusarium wilt disease of cucumber plants with the application of a bioorganic fertilizer. Biol. Fertil. Soils 44:1073-1080.

Zhou, T., Chen, D., Li, C., Sun, Q., Li, L., Liu, F., Shen, Q., and Shen, B. 2012. Isolation and characterization of Pseudomonas brassicacearum $\mathrm{J} 12$ as an antagonist against Ralstonia solanacearum and identification of its antimicrobial components. Microbiol. Res. 167:388-394. 